PROCEEDINGS OF THE

AMERICAN MATHEMATICAL SOCIETY

Volume 128, Number 10, Pages 2957-2965

S 0002-9939(00)05838-X

Article electronically published on June 6, 2000

\title{
FIXED POINT RESULTS FOR GENERALIZED CONTRACTIONS IN GAUGE SPACES AND APPLICATIONS
}

\author{
M. FRIGON
}

(Communicated by David R. Larson)

\begin{abstract}
In this paper, we present fixed point results for generalized contractions defined on a complete gauge space $\mathbb{E}$. Also, we consider families of generalized contractions $\left\{f^{t}: X \rightarrow \mathbb{E}\right\}_{t \in[0,1]}$ where $X \subset \mathbb{E}$ is closed and can have empty interior. We give conditions under which the existence of a fixed point for some $f^{t_{0}}$ imply the existence of a fixed point for every $f^{t}$. Finally, we apply those results to infinite systems of first order nonlinear differential equations and to integral equations on the real line.
\end{abstract}

\section{IntRoduCtion}

A fundamental result in fixed point theory is the well-known Banach Contraction Principle which states that every contraction on a complete metric space into itself has a unique fixed point.

In 1971, Cain and Nashed [1 extended the notion of contraction to Hausdorff locally convex linear spaces $\left(\mathbb{E},\left\{|\cdot|_{\alpha}\right\}_{\alpha \in \Lambda}\right.$ ) (where $\left\{|\cdot|_{\alpha}\right\}_{\alpha \in \Lambda}$ is a family of seminorms) as follows: let $X \subset \mathbb{E}$, a function $f: X \rightarrow X$ is a contraction if for every $\alpha \in \Lambda$, there exists $k_{\alpha}<1$ such that

$$
|f(x)-f(y)|_{\alpha} \leq k_{\alpha}|x-y|_{\alpha} \quad \text { for every } x, y \in X .
$$

They showed that the Banach Contraction Principle is still valid for contraction defined on sequentially complete subset $X$.

Also, the notion of contraction has been extended to uniform spaces (see [8], [13], [14]) and to the condensing map on Hausdorff locally convex linear spaces via the notion of measure of noncompactness (see [15], [16]).

However, in most of those extensions, $f$ satisfies a restrictive condition of the form:

$$
\text { if }|x-y|_{\alpha}=0, \quad \text { then }|f(x)-f(y)|_{\alpha}=0 .
$$

For example, consider the particular case where $\mathbb{E}=\prod_{n \in \mathbb{N}} E_{n}$ with $\left(E_{n},|\cdot|_{n}\right)$ Banach spaces, $X \subset \mathbb{E}$, and $f=\left(f_{1}, f_{2}, \ldots\right): X \rightarrow X$ is such that there exists a matrix $A=\left(a_{i j}\right)$ with nonnegative entries such that for every $n \in \mathbb{N}$,

$$
\left|f_{n}(x)-f_{n}(y)\right|_{n} \leq a_{n 1}\left|x_{1}-y_{1}\right|_{1}+a_{n 2}\left|x_{2}-y_{2}\right|_{2}+\cdots .
$$

Received by the editors November 19, 1998.

1991 Mathematics Subject Classification. Primary 47H10, 47N20.

This work was partially supported by CRSNG Canada. 
If $f$ is a contraction, we can choose the matrix $A$ lower triangular. In other words, $f_{n}$ does not depend on $x_{n+1}, x_{n+2}, \ldots$.

In this paper, in introducing the notion of generalized contraction, we allow, in this particular context, $a_{i j}>0$ for every $i, j \in \mathbb{N}$.

More precisely, for $\mathbb{E}$ a complete gauge space, we establish in section 3 that a generalized contraction $f: \mathbb{E} \rightarrow \mathbb{E}$ has a unique fixed point. Let us point out that $f$ does not satisfy a condition analogous to (1.1). Our fixed point theorem extends results of Cain and Nashed [1, and Tarafdar [13.

Then, in section 4, we consider suitable families of generalized contractions $\left\{f^{t}: X \rightarrow \mathbb{E}\right\}_{t \in[0,1]}$ with $X$ a closed subset of $\mathbb{E}$. We give assumptions under which the existence of a fixed point for some $f^{t_{0}}$ imply the existence of a fixed point for every $f^{t}$. It is worthwhile to notice that, here, $X$ can have empty interior. Our results extend those of [5]. Moreover, even when $\mathbb{E}$ is a locally convex space, they can not be obtained from the topological degree theory for condensing maps (see [15, [16, [17]). Our proofs rely on a fixed point theorem of Frigon and Granas [4] for multivalued contractions.

In the last section, we present some examples of applications of our fixed point results. The first one is a generalization of Picard's Theorem to infinite systems of first order differential equations:

$$
\begin{aligned}
& x^{\prime}(t)=g(t, x(t)), \quad t \in[0, T], \\
& x(0)=0,
\end{aligned}
$$

where $x=\left(x_{1}, x_{2}, \ldots\right), g=\left(g_{1}, g_{2}, \ldots\right)$, and there exists a matrix $A=\left(a_{i j}\right)$ with nonnegative entries such that

$$
\left(\begin{array}{c}
\left\|g_{1}(t, x)-g_{1}(t, y)\right\|_{1} \\
\left\|g_{2}(t, x)-g_{2}(t, y)\right\|_{2} \\
\vdots
\end{array}\right) \leq A\left(\begin{array}{c}
\left\|x_{1}-y_{1}\right\|_{1} \\
\left\|x_{2}-y_{2}\right\|_{2} \\
\vdots
\end{array}\right)
$$

Among mathematicians who treated this problem, let us mention Dore 2] who used the measure of noncompactness, and Moszyński and Pokrzywa [1] who assume the existence of $A e^{A T}$. Considerable works on this problem in the case where $A$ is row-finite were done by Herzog, Lemmert, Weckbach (see 6], 7], 9], [10] and the references therein). Their results were mainly obtained for $A$ with at most countable spectrum, or equivalently when $e^{A t}$ exists. Also, the solution to (1.2) is obtained by the method of successive approximation.

Our results are independent of those mentionned above. We do not assume that $A$ is row-finite. In fact, we can have $a_{i j}>0$ for every $i, j \in \mathbb{N}$. Also, the existence of a solution to (1.2) follows from a fixed point result, not from an iteration process.

Finally, an example of application to integral equation on the real line is presented.

\section{Notations}

Let $\mathbb{E}$ be a gauge space endowed with a complete gauge structure $\left\{d_{\alpha}: \alpha \in \Lambda\right\}$, where $\Lambda$ is a directed set (see [3] for definitions). We will assume that the following condition is satisfied:

$$
d_{\alpha}(x, y) \leq d_{\beta}(x, y) \text { for every } x, y \in E \text { and every } \alpha \leq \beta .
$$


To $\mathbb{E}$, we associate for every $\alpha \in \Lambda$ a metric space $\left(\mathbb{E}_{\alpha}, d_{\alpha}\right)$ as follows: for each $\alpha \in \Lambda$, we write

$$
x \sim_{\alpha} y \quad \text { if and only if } \quad d_{\alpha}(x, y)=0 .
$$

This defines an equivalence relation on $\mathbb{E}$. We denote by $E_{\alpha}=\left(E / \sim_{\alpha}, d_{\alpha}\right)$ the quotient space, and by $\left(\mathbb{E}_{\alpha}, d_{\alpha}\right)$ the completion of $E_{\alpha}$ with respect to $d_{\alpha}$ (the metric on $E_{\alpha}$ induced by $d_{\alpha}$ and its extention to $\mathbb{E}_{\alpha}$ are still denoted by $d_{\alpha}$ ). This construction defines a continuous map $\mu_{\alpha}: \mathbb{E} \rightarrow \mathbb{E}_{\alpha}$.

Since the condition $(\star)$ is satisfied, the pseudometric $d_{\alpha}$ induces a pseudometric on $\mathbb{E}_{\beta}$ for every $\beta \geq \alpha$. For simplicity, the pseudometric is still denoted by $d_{\alpha}$. Again, the relation (2.1) defines an equivalence relation on $\mathbb{E}_{\beta}$ from which we obtain a continuous map $\mu_{\alpha \beta}: \mathbb{E}_{\beta} \rightarrow \mathbb{E}_{\alpha}$ since $\mathbb{E}_{\beta} / \sim_{\alpha}$ can be regarded as a subset of $\mathbb{E}_{\alpha}$. Observe that $\mathbb{E}$ is the projective limit of $\left(\mathbb{E}_{\alpha}\right)_{\alpha \in \Lambda}$.

For each subset $X \subset \mathbb{E}$ and each $\alpha \in \Lambda$, we set $X_{\alpha}=\mu_{\alpha}(X)$, and we denote by $\overline{X_{\alpha}}$ and $\partial_{\alpha} X_{\alpha}$, respectively, the closure and the boundary of $X_{\alpha}$ with respect to $d_{\alpha}$ in $\mathbb{E}_{\alpha}$. Notice that $\mu_{\alpha \beta}\left(X_{\beta}\right) \subset X_{\alpha}$.

The following lemma gives an important property of closed subsets of $\mathbb{E}$.

Lemma 2.1. Assume that the condition $(\star)$ is satisfied, and let $X$ be a closed subset of $\mathbb{E}$. Then, for every net $\left(z_{\alpha}\right)_{\alpha \in \Lambda}$ with $z_{\alpha} \in \overline{X_{\alpha}}$, such that for every $\alpha \in \Lambda$, $\left(\mu_{\alpha \beta}\left(z_{\beta}\right)\right)_{\beta \geq \alpha}$ is a Cauchy net in $\overline{X_{\alpha}}$, there exists $x \in X$ such that $\left(\mu_{\alpha \beta}\left(z_{\beta}\right)\right)_{\beta \geq \alpha}$ converges to $\mu_{\alpha}(x) \in X_{\alpha}$ for every $\alpha \in \Lambda$.

We denote by $\operatorname{diam}_{\alpha}$ and $D_{\alpha}$ the $\alpha$-diameter and the generalized Hausdorff pseudometric induced by $d_{\alpha}$; that is, for $X, Y \subset \mathbb{E}$,

$$
\begin{gathered}
\operatorname{diam}_{\alpha}(X)=\sup \left\{d_{\alpha}(x, \hat{x}): x, \hat{x} \in X\right\}, \\
D_{\alpha}(X, Y)=\inf \{\varepsilon>0: \forall x \in X, \forall y \in Y, \exists \hat{x} \in X, \exists \hat{y} \in Y \text { such that } \\
\left.\qquad d_{\alpha}(x, \hat{y})<\varepsilon, d_{\alpha}(\hat{x}, y)<\varepsilon\right\},
\end{gathered}
$$

with the convention that $\inf (\emptyset)=\infty$. We will also denote $\operatorname{diam}_{\alpha}$ and $D_{\alpha}$, the diameter and the generalized Hausdorff metric induced by $d_{\alpha}$ in $\mathbb{E}_{\alpha}$. In the particular case where $\mathbb{E}$ is a locally convex linear space, we say that a subset $X \subset \mathbb{E}$ is bounded if $D_{\alpha}(\{0\}, X)<\infty$ for every $\alpha \in \Lambda$.

Let $f: X \rightarrow \mathbb{E}$ be a function. For every $\alpha \in \Lambda$, we define a multivalued function $F_{\alpha}: X_{\alpha} \rightarrow \mathbb{E}_{\alpha}$ by

$$
F_{\alpha}\left(\mu_{\alpha}(x)\right)=\overline{\mu_{\alpha} \circ f\left(\{x\}_{\alpha}\right)},
$$

where $\{x\}_{\alpha}=\left\{y \in X: d_{\alpha}(x, y)=0\right\}$. When it exists, the multivalued continuous extension of $F_{\alpha}$ with closed values will be denoted by

$$
\mathbb{F}_{\alpha}: \overline{X_{\alpha}} \rightarrow \mathbb{E}_{\alpha}
$$

Finally, if $A=\left(a_{i j}\right)$ is a matrix with nonnegative real entries, we denote

$$
a_{n}=\max _{1 \leq i \leq n} \sum_{j=1}^{n} a_{i j} \quad \text { and } \quad \alpha_{n}=\max _{1 \leq i \leq n} \sum_{j=n+1}^{\infty} a_{i j}
$$




\section{Generalized contractions}

We introduce the notion of generalized contraction on a subset $X$ of $\mathbb{E}$ a complete gauge space.

Definition 3.1. A function $f: X \rightarrow \mathbb{E}$ is a generalized contraction if

(i) for every $\alpha \in \Lambda$, there exists $k_{\alpha}<1$ such that

$$
D_{\alpha}\left(f\left(\{x\}_{\alpha}\right), f\left(\{y\}_{\alpha}\right)\right) \leq k_{\alpha} d_{\alpha}(x, y) \quad \text { for all } x, y \in X
$$

(ii) for every $\varepsilon>0$ and every $\alpha \in \Lambda$, there exists $\beta \geq \alpha$ such that

$$
\operatorname{diam}_{\beta}\left(f\left(\{x\}_{\beta}\right)\right)<\left(1-k_{\beta}\right) \varepsilon \text { for every } x \in X .
$$

We will also say that $f$ is a generalized contraction with constants $\left\{k_{\alpha}\right\}$.

Remark 3.2. It is obvious that a contraction $f: X \rightarrow \mathbb{E}$ is a generalized contraction since $\operatorname{diam}_{\alpha}\left(f\left(\{x\}_{\alpha}\right)\right)=0$ for every $x \in X$. Also, if $\Lambda=\mathbb{N}$, notice that $f$ can be a generalized contraction without being a contraction in the classical sense with the metric $d(x, y)=\sum_{n \in \mathbb{N}} d_{n}(x, y) / 2^{n}\left(1+d_{n}(x, y)\right)$ on $\mathbb{E}$.

We present a generalization of the Banach Contraction Principle.

Theorem 3.3. Let $\mathbb{E}$ be a complete gauge space. Every generalized contraction $f: \mathbb{E} \rightarrow \mathbb{E}$ has a unique fixed point.

Proof. Since $f$ is a generalized contraction, for every $\alpha \in \Lambda$, the continuous extension introduced in (2.2), $\mathbb{F}_{\alpha}: \mathbb{E}_{\alpha} \rightarrow \mathbb{E}_{\alpha}$ exists. Moreover, it is a multivalued contraction with closed values in a complete metric space. By the Nadler Fixed Point Theorem (see [12] or [4]), it has a fixed point $z_{\alpha} \in \mathbb{F}_{\alpha}\left(z_{\alpha}\right)$. Obviously, $\mu_{\alpha \beta}\left(z_{\beta}\right) \in \mathbb{F}_{\alpha}\left(\mu_{\alpha \beta}\left(z_{\beta}\right)\right)$ for all $\beta \geq \alpha$.

Let $\varepsilon>0$ and $\gamma \in \Lambda$. Since $f$ is a generalized contraction, there exists $\alpha \geq \gamma$ such that

$$
\operatorname{diam}_{\alpha}\left(\mathbb{F}_{\alpha}(z)\right)<\left(1-k_{\alpha}\right) \varepsilon \text { for all } z \in \mathbb{E}_{\alpha} .
$$

It follows that for $\lambda, \beta \geq \alpha$,

$$
\begin{aligned}
d_{\alpha}\left(\mu_{\alpha \lambda}\left(z_{\lambda}\right), \mu_{\alpha \beta}\left(z_{\beta}\right)\right) & \leq D_{\alpha}\left(\mathbb{F}_{\alpha}\left(\mu_{\alpha \lambda}\left(z_{\lambda}\right)\right), \mathbb{F}_{\alpha}\left(\mu_{\alpha \beta}\left(z_{\beta}\right)\right)\right)+\operatorname{diam}_{\alpha}\left(\mathbb{F}_{\alpha}\left(\mu_{\alpha \lambda}\left(z_{\lambda}\right)\right)\right) \\
& <k_{\alpha} d_{\alpha}\left(\mu_{\alpha \lambda}\left(z_{\lambda}\right), \mu_{\alpha \beta}\left(z_{\beta}\right)\right)+\left(1-k_{\alpha}\right) \varepsilon
\end{aligned}
$$

Hence,

$$
d_{\gamma}\left(\mu_{\gamma \lambda}\left(z_{\lambda}\right), \mu_{\gamma \beta}\left(z_{\beta}\right)\right) \leq d_{\alpha}\left(\mu_{\alpha \lambda}\left(z_{\lambda}\right), \mu_{\alpha \beta}\left(z_{\beta}\right)\right)<\varepsilon .
$$

So, for all $\gamma \in \Lambda,\left(\mu_{\gamma \alpha}\left(z_{\alpha}\right)\right)_{\alpha \geq \gamma}$ is a Cauchy net in $\mathbb{E}_{\gamma}$, and therefore, converges in the sense of Lemma 2.1 to some $x \in \mathbb{E}$.

We claim that $x=f(x)$. Indeed, let $\gamma \in \Lambda$. For $\varepsilon>0$, choose $\beta \geq \alpha \geq \gamma$ such that $\operatorname{diam}_{\alpha}\left(f\left(\{x\}_{\alpha}\right)\right)<\varepsilon / 2$ and $d_{\alpha}\left(\mu_{\alpha}(x), \mu_{\alpha \beta}\left(z_{\beta}\right)\right)<\varepsilon / 4$. We have

$$
\begin{aligned}
d_{\gamma}(x, f(x)) \leq & d_{\alpha}(x, f(x)) \\
\leq & d_{\alpha}\left(\mu_{\alpha}(x), \mu_{\alpha \beta}\left(z_{\beta}\right)\right)+D_{\alpha}\left(\mathbb{F}_{\alpha}\left(\mu_{\alpha \beta}\left(z_{\beta}\right)\right), \mathbb{F}_{\alpha}\left(\mu_{\alpha}(x)\right)\right) \\
& +\operatorname{diam}_{\alpha}\left(\mathbb{F}_{\alpha}\left(\mu_{\alpha}(x)\right)\right) \\
\leq & \left(1+k_{\alpha}\right) d_{\alpha}\left(\mu_{\alpha}(x), \mu_{\alpha \beta}\left(z_{\beta}\right)\right)+\frac{\varepsilon}{2} \leq \varepsilon .
\end{aligned}
$$

A similar argument permits us to deduce the uniqueness of $x$ the fixed point of $f$. 
Remark 3.4. It is an open question to know if we can define inductively a sequence converging to the fixed point of $f$.

Corollary 3.5. Let $\mathbb{E}=\prod_{n \in \mathbb{N}} E_{n}$ and $B=\prod_{n \in \mathbb{N}} B_{n}$, where $\left(E_{n},\|\cdot\|_{n}\right)$ is a Banach space and $B_{n}$ is the closed unit ball in $E_{n}$. Assume that $f=\left(f_{1}, f_{2}, \ldots\right)$ : $B \rightarrow B$ is a function such that

$$
\left(\begin{array}{c}
\left\|f_{1}(x)-f_{1}(y)\right\|_{1} \\
\left\|f_{2}(x)-f_{2}(y)\right\|_{2} \\
\vdots
\end{array}\right) \leq A\left(\begin{array}{c}
\left\|x_{1}-y_{1}\right\|_{1} \\
\left\|x_{2}-y_{2}\right\|_{2} \\
\vdots
\end{array}\right),
$$

for $A=\left(a_{i j}\right)$ a matrix with nonnegative entries such that for every $n \in \mathbb{N}$,

$$
a_{n}<1 \quad \text { and } \quad \liminf _{n \rightarrow \infty} \frac{\alpha_{n}}{1-a_{n}}=0,
$$

where $a_{n}$ and $\alpha_{n}$ are defined in (2.3). Then $f$ has a unique fixed point.

Proof. For $n \in \mathbb{N}$, we define the semi-norm $|x|_{n}=\max \left\{\left\|x_{1}\right\|_{1}, \ldots,\left\|x_{n}\right\|_{n}\right\}$. For $x$, $y \in B$, we have $\left|f(x)-f\left(y_{1}, \ldots, y_{n}, x_{n+1}, \ldots\right)\right|_{n} \leq a_{n}|x-y|_{n}$. Thus,

$$
D_{n}\left(f\left(\{x\}_{n}\right), f\left(\{y\}_{n}\right)\right) \leq a_{n}|x-y|_{n} .
$$

Moreover,

$$
\operatorname{diam}_{n}\left(f\left(\{x\}_{n}\right)\right) \leq 2 \alpha_{n}
$$

So, the function $f$ is a generalized contraction, and the conclusion follows from Theorem [3.3.

Remark 3.6. We can replace $B$ by a bounded subset of $\mathbb{E}$ in modifying the definition of $\alpha_{n}$.

\section{Families of Generalized CONTRACtions}

In this paragraph, we consider families of generalized contractions depending on a parameter $\left\{f^{t}: X \rightarrow \mathbb{E}\right\}$ with $X \subset \mathbb{E}$ closed. We want to give assumptions under which the existence of a fixed point for some $f^{t_{0}}$ implies the existence of a fixed point for each $f^{t}$.

Definition 4.1. A family of generalized contractions $\left\{f^{t}: X \rightarrow \mathbb{E}\right\}_{t \in[0,1]}$ is said to be admissible if:

(i) there exists $\left\{k_{\alpha}\right\}_{\alpha \in \Lambda}$ with $k_{\alpha}<1$ for all $\alpha \in \Lambda$, such that for every $t \in[0,1]$, $f^{t}$ is a generalized contraction with constants $\left\{k_{\alpha}\right\}$;

(ii) for all $\alpha \in \Lambda$, there exists $M_{\alpha} \geq 0$ such that for every $x \in X$, and every $t$, $s \in[0,1], D_{\alpha}\left(f^{t}\left(\{x\}_{\alpha}\right), f^{s}\left(\{x\}_{\alpha}\right)\right) \leq M_{\alpha}|t-s|$

(iii) $z \notin \mathbb{F}_{\alpha}^{t}(z)$ for every $z \in \partial X_{\alpha}, t \in[0,1], \alpha \in \Lambda$.

Remark 4.2. Since $f^{t}$ is a generalized contraction, $\mathbb{F}_{\alpha}^{t}$ exists. On the other hand, it is suitable to impose condition (iii) instead of

(iii) $^{\prime} x \neq f^{t}(x)$ for every $x \in \partial X, t \in[0,1]$.

Indeed, in many applications, $X$ has empty interior: in this case, (iii) ${ }^{\prime}$ means that $f^{t}$ has no fixed point for every $t$.

Theorem 4.3. Let $X$ be a closed subset of $\mathbb{E}$, and let $\left\{f^{t}: X \rightarrow \mathbb{E}\right\}_{t \in[0,1]}$ be an admissible family of generalized contractions. If $f^{t}$ has a fixed point for some $t \in[0,1]$, then $f^{t}$ has a unique fixed point for all $t \in[0,1]$. 
Proof. If $f^{t_{0}}$ has a fixed point, then $\mathbb{F}_{\alpha}^{t_{0}}$ has also a fixed point for all $\alpha \in \Lambda$. The map defined on $\overline{X_{\alpha}} \times[0,1]$ by $(z, t) \mapsto \mathbb{F}_{\alpha}^{t}(z) \in \mathbb{E}_{\alpha}$ satisfies the assumptions of Theorem 4.3 in [4, which gives for each $t \in[0,1]$ and each $\alpha \in \Lambda$ the existence of $z_{\alpha}^{t} \in \overline{X_{\alpha}}$ such that $z_{\alpha}^{t} \in \mathbb{F}_{\alpha}^{t}\left(z_{\alpha}^{t}\right)$.

In using the fact that for every $t \in[0,1], f^{t}$ is a generalized contraction, and in arguing as in Theorem 3.3 we show that the net $\left(z_{\alpha}^{t}\right)_{\alpha \in \Lambda}$ converges in the sense of Lemma 2.1 to some $x^{t} \in X$ such that $x^{t}=f^{t}\left(x^{t}\right)$.

From the previous theorem, we get the following results.

Corollary 4.4. Let $X$ be a closed subset of $\mathbb{E}$, a complete Hausdorff locally convex linear space such that $0 \in X$. Let $f: X \rightarrow \mathbb{E}$ be a generalized contraction such that $f(X)$ is bounded. Then one of the following statements holds:

(a) $f$ has a unique fixed point;

(b) there exist $t \in\left[0,1\left[, \alpha \in \Lambda\right.\right.$, and $z \in \partial_{\alpha} X_{\alpha}$ such that $z \in t \mathbb{F}(z)$.

Corollary 4.5. Let $\mathbb{E}, B$, and $f: B \rightarrow \mathbb{E}$ be as in Corollary [3.5. Then one of the following statements holds:

(a) $f$ has a unique fixed point;

(b) there exist $n \in \mathbb{N}, t \in] 0,1\left[\right.$, and $x=\left(x_{1}, x_{2}, \ldots\right) \in B$ such that $\left\|x_{n}\right\|_{n}=1$ and $\left\|x_{n}-t f_{n}(x)\right\|_{n}=0$.

\section{Applications}

In this section, we present examples of applications of the previous fixed point results. For sake of simplicity, we chose to not be as general as possible.

First of all, we present a generalization of the Picard Theorem to infinite systems of first order differential equations

$$
\begin{aligned}
& x^{\prime}(t)=g(t, x(t)), \quad t \in[0, T], \\
& x(0)=0,
\end{aligned}
$$

where $x=\left(x_{1}, x_{2}, \ldots\right)$ and $g=\left(g_{1}, g_{2}, \ldots\right)$.

Theorem 5.1. Let $E=\prod_{n \in \mathbb{N}} E_{n}$ with $\left(E_{n},\|\cdot\|_{n}\right)$ be Banach spaces, and $B=$ $\prod_{n \in \mathbb{N}} B_{n}$. Assume that $g:[0, \tau] \times B \rightarrow E$ is a continuous function such that

(a) $M=\sup \left\{\left\|g_{n}(t, x)\right\|_{n}: x \in B, t \in[0, \tau], n \in \mathbb{N}\right\}<\infty$;

(b) there exists a matrix $A=\left(a_{i j}\right)$ with nonnegative entries such that

$$
\left(\begin{array}{c}
\left\|g_{1}(t, x)-g_{1}(t, y)\right\|_{1} \\
\left\|g_{2}(t, x)-g_{2}(t, y)\right\|_{2} \\
\vdots
\end{array}\right) \leq A\left(\begin{array}{c}
\left\|x_{1}-y_{1}\right\|_{1} \\
\left\|x_{2}-y_{2}\right\|_{2} \\
\vdots
\end{array}\right)
$$

and

$$
\liminf _{n \rightarrow \infty} \alpha_{n} a_{n}^{-1} e^{a_{n} / M}=0
$$

Then the system (5.1) has a unique solution in $\prod_{n \in \mathbb{N}} C^{1}\left([0, T], E_{n}\right)$ with $T=$ $\min \{\tau, 1 / M\}$. 
Proof. Denote $\mathbb{E}=\prod_{n \in \mathbb{N}} C\left([0, T], E_{n}\right), X=\prod_{n \in \mathbb{N}} C\left([0, T], B_{n}\right)$, and define $f: X \rightarrow X$ by

$$
f(x)(t)=\int_{0}^{t} g(s, x(s)) d s .
$$

The assumption (a) and the definition of $T$ imply that the function $f$ is well defined. Observe that a solution to the system is a fixed point of $f$.

We introduce on $\mathbb{E}$ the family of semi-norm $\left\{|\cdot|_{n}\right\}_{n \in \mathbb{N}}$, where

$$
|x|_{n}=\max _{t \in[0, T]}\left\{e^{a_{n}(T-t)} \max \left\{\left\|x_{1}(t)\right\|_{1}, \ldots,\left\|x_{n}(t)\right\|_{n}\right\}\right\} .
$$

It is easy to show that

$$
D_{n}\left(f\left(\{x\}_{n}\right), f\left(\{y\}_{n}\right)\right) \leq\left(1-e^{-a_{n} T}\right)|x-y|_{n} \quad \text { for all } x, y \in X
$$

and

$$
\operatorname{diam}_{n}\left(f\left(\{x\}_{n}\right)\right) \leq 2 \alpha_{n} \max \left\{T, a_{n}^{-1} e^{a_{n} T}\right\} \quad \text { for all } x \in X .
$$

The conclusion follows from Theorem 3.3 .

Remark 5.2. (i) For simplicity, we stated the previous result for the homogenous initial condition and for a function defined on a product of closed unit ball. It is obvious that with a slight modification of the assumptions, an analogous result can be obtained for the nonhomogenous initial condition $x(0)=\left(\bar{x}_{1}, \bar{x}_{2}, \ldots\right)$, and $g$ defined on the product of $B_{n}\left(\bar{x}_{n}, r_{n}\right)$ the closed ball centered in $\bar{x}_{n}$ of radius $r_{n}$.

(ii) Here, each function $\left(x_{1}, x_{2}, \ldots\right) \mapsto g_{i}\left(t, x_{1}, x_{2}, \ldots\right)$ can really depend on an infinite number of $x_{n}$. This is not the case for most of the results of the literature; for example in 7], [10, where the matrix $A$ must be row-finite.

The next result shows that with a better knowledge of the growth of $g$, we can improve the interval where we get the existence of a solution.

Theorem 5.3. Let $g:[0, \infty[\times B \rightarrow E$ be a continuous function with $B$ and $E$ as in the previous theorem. Assume that

(a) for every $n \in \mathbb{N}$, there exist $b_{n} \geq 0, m_{n} \in L_{\text {loc }}^{1}[0, \infty[$ such that

$$
\||| g(t, x)\|\left\|_{n} \leq m_{n}(t)+b_{n}\right\||| x \mid \|_{n} \quad \text { for every } t \in[0, \infty[, x \in E,
$$

and

$$
\left.\sup \left\{t: M_{n}(t) e^{b_{n} t} \leq 1\right\}=T \in\right] 0, \infty[,
$$

where $M_{n}(t)=\int_{0}^{t} m_{n}(s) d s,\left\|\left|\|x \mid\|_{n}=\max \left\{\left\|x_{1}\right\|_{1}, \ldots,\left\|x_{n}\right\|_{n}\right\} ;\right.\right.$

(b) $g$ satisfies the assumption (b) of the previous theorem with $M=1 / T$.

Then, system (5.1) has a unique solution in $\prod_{n \in \mathbb{N}} C^{1}\left([0, T], E_{n}\right)$.

Proof. Let $\mathbb{E}, X$, and $f: X \rightarrow \mathbb{E}$ be as in the proof of the previous theorem. Again, $f$ is a generalized contraction.

On the other hand, if $z \in \prod_{i=1}^{n} C\left([0, T], B_{i}\right)$ is such that $z \in \mu \mathbb{F}_{n}(z)$ for some $\mu \in[0,1[$, then

$$
\left\||| z(t)\left|\left\|_{n} \leq \mu \int_{0}^{t} m_{n}(s)+b_{n}(s)\right\|\right| z(s) \mid\right\|_{n} d s \quad \text { for every } t \in[0, T] .
$$


In using Gronwall's inequality and assumption (a), we deduce that

$$
|| z(t) \mid \|_{n} \leq \mu \int_{0}^{t} m(s) e^{b_{n}(t-s)} d s<1 \quad \text { for every } t \in[0, T] .
$$

The conclusion follows from Corollary 4.4,

Now, we give an application to integral equations in Banach spaces

$$
x(t)=\int_{\mathbb{R}} g(t, s, x(s)) d s .
$$

Theorem 5.4. Let $(E,\|\|)$ be a Banach space, and let $g: \mathbb{R}^{2} \times E \rightarrow E$ be a continuous function. Assume that

(a) there exists a continuous function $k:[0, \infty[\times \mathbb{R} \rightarrow[0, \infty)$ nondecreasing in its first argument such that

$$
\|g(t, s, x)-g(t, s, y)\| \leq k(|t|, s)\|x-y\| \text { for } s, t \in \mathbb{R}, x, y \in E,
$$

and

$$
\|k(t, \cdot)\|_{L^{1}\left(I_{t}\right)}=1-h_{t}<1 \quad \text { for every } t \in[0, \infty[,
$$

where $I_{t}=[-t, t]$;

(b) there exists a function $\phi:[0, \infty) \times \mathbb{R} \rightarrow[0, \infty)$ nondecreasing in its first argument such that for every $t \geq 0, \phi(t, \cdot) \in L^{1}\left(I_{t}^{c}\right)$,

$$
\|g( \pm t, s, x)\| \leq \phi(t, s) \quad \text { for } s \in I_{t}^{c}, x \in E
$$

and

$$
\liminf _{t \rightarrow \infty} h_{t}^{-1}\|\phi(t, \cdot)\|_{L^{1}\left(I_{t}^{c}\right)}=0 .
$$

Then the integral equation (5.2) has a unique solution in $C(\mathbb{R}, E)$.

Proof. We set $\mathbb{E}=C(\mathbb{R}, E)$, endowed with the family of semi-norms $\left\{|\cdot|_{t}\right\}_{t \in[0, \infty[}$, where

$$
|x|_{t}=\sup \{\|x(\tau)\|: \tau \in[-t, t]\} .
$$

We consider $f: \mathbb{E} \rightarrow \mathbb{E}$ the function defined by

$$
f(x)(t)=\int_{\mathbb{R}} g(t, s, x(s)) d s .
$$

The function $f$ is a generalized contraction. Indeed, fix $t \in[0, \infty[$, and take $x$ and $y \in \mathbb{E}$. The assumption (a) implies the existence of a sequence $\left(x_{j}\right)$ in $\mathbb{E}$ such that $\left.x_{j}\right|_{\{-t, t\}}=\left.y\right|_{\{-t, t\}}$, and

$$
\int_{I_{t}^{c}} g(\tau, s, x(s))-g\left(\tau, s, x_{j}(s)\right) d s \rightarrow 0
$$

uniformly for $\tau \in I_{t}$. Thus, for $\varepsilon>0$, there exists $j$ sufficiently large such that for all $\tau \in I_{t}$,

$$
\begin{aligned}
& \left\|\int_{\mathbb{R}} g(\tau, s, x(s)) d s-\int_{I_{t}} g(\tau, s, y(s)) d s-\int_{I_{t}^{c}} g\left(\tau, s, x_{j}(s)\right) d s\right\| \\
& \quad \leq \int_{I_{t}} k(t, s)\|x(s)-y(s)\| d s+\left\|\int_{I_{t}^{c}} g(\tau, s, x(s))-g\left(\tau, s, x_{j}(s)\right) d s\right\| \\
& \quad \leq\left(1-h_{t}\right)|x-y|_{t}+\varepsilon .
\end{aligned}
$$


Therefore,

$$
D_{t}\left(f\left(\{x\}_{t}\right), f\left(\{y\}_{t}\right)\right) \leq\left(1-h_{t}\right)|x-y|_{t} .
$$

In using condition (b), we show that

$$
\sup _{x \in \mathbb{E}} h_{t}^{-1} \operatorname{diam}_{t}\left(f\left(\{x\}_{t}\right)\right) \rightarrow 0 .
$$

The conclusion follows from Theorem 3.3 ,

\section{REFERENCES}

[1] G. L. Cain JR. And M. Z. NAshed, Fixed points and stability for a sum of two operators in locally convex spaces, Pacific J. Math. 39 (1971), 581-592. MR 48:968

[2] G. Dore, Sul problema di Cauchy per equazioni differenziali ordinarie in spazi localmente convessi, Rend. Mat. Appl. (7) 1 (1981), 237-247.

[3] J. Dugundur. Topology, Wm. C. Brown Publ., Dubuque, 1989.

[4] M. Frigon and A. Granas, Résultats du type Leray-Schauder pour des contractions multivoques, Topol. Methods Nonlinear Anal. 4 (1994), 197-208. MR 95m:47110

[5] M. Frigon and A. Granas, Résultats de type Leray-Schauder pour des contractions sur des espaces de Fréchet, Ann. Sci. Math. Québec 22 (1998), 161-168. CMP 99:09

[6] G. Herzog, On ordinary linear differential equations in $\mathbb{C}^{J}$, Demonstratio Math. 28 (1995), 383-398.

[7] G. Herzog, On Lipschitz conditions for ordinary differential equations in Fréchet spaces, Czechoslovak Math. J. 48 (1998), 95-103. MR 99c:34134

[8] R. J. Knill, Fixed points of uniform contractions, J. Math. Anal. Appl. 12 (1965), 449-455. MR 33:709

[9] R. LEMmert AND Ä. WECKBACH, Charakterisierung zeilenendlicher Matrizen mit abzählbarem Spektrum, Math. Z. 188 (1984), 119-124. MR 87h:47011

[10] R. Lemmert, On ordinary differential equations in locally convex spaces, Nonlinear Anal. 10 (1986), 1385-1390. MR 88c:34084

[11] K. Moszyński And A. Pokrzywa, Sur les systèmes infinis d'équations différentielles ordinaires dans certains espaces de Fréchet, Dissertationes Math. (Rozprawy Mat.) 115 (1974), 1-32. MR 52:11241

[12] S. B. NADLER JR., Multi-valued contraction mappings, Pacific J. Math. 30 (1969), 415-487. MR 40:8035

[13] E. TARAfdar, An approach to fixed-point theorems on uniform spaces, Trans. Amer. Math. Soc. 191 (1974), 209-225. MR 50:14725

[14] W. W. TAYLOR, Fixed points theorems for nonexpansive mappings in linear topological spaces, J. Math. Anal. Appl. 40 (1972), 164-173. MR 48:974

[15] B. N. Sadovskĭ, Measures of noncompactness and condensing operators, Probl. Mat. Anal. 2 (1968), 88-119 (Russian). MR 46:740

[16] B. N. Sadovskǐ, Limit compact and condensing operators, Uspekhi Mat. Nauk 27 (1972), 81-146 (Russian). MR 55:1161

[17] T. Sengadir, D. V. Pai, And A. K. Pani, A Leray-Schauder type theorem and applications to boundary value problems for neutral equations, Nonlinear Anal. 28 (1997), 701-719. MR 98b: 47072

Département de Mathématiques et Statistique, Université de Montréal, C. P. 6128, Succ. Centre-ville, Montréal, Canada H3C 3J7

E-mail address: frigon@dms.umontreal.ca 